\title{
ESTIMATION OF OPTIMAL PATH ON URBAN ROAD NETWORKS USING AHP ALGORITHM
}

\author{
Surendra Kukadapwar ${ }^{1}$, Dhananjay Parbat ${ }^{2}$ \\ ${ }^{1,2}$ Department of Civil Engineering, Government Polytechnic, Nagpur, India
}

Received 14 September 2015; accepted 29 January 2016

\begin{abstract}
This paper describes to develop a multi criteria decision based methodology to find optimal path in real urban road network. Over the year several studies were conducted but most of which rely on single variable like travel distance or travel time as cost function. In this study, seven different attributes influencing the traffic network i.e. distance, time, traffic volume, road width, no. of intersection, parking and encroachment on road are used to define cost function using multi criterion decision making approach. These variables are combined using a Multi-Dimensional Cost Model (MDCM) using the Analytical Hierarchical Process (AHP). The models developed were implemented and closely evaluated in Nagpur city of India. Model is considered for determining optimal path between various Origins and Destinations in real urban traffic network. Composite weighted AHP scored were used to generate AHP decision surface. Finally, the best decision was proposed by generating the least cost path which is considered as optimal path. The resulting routes showed to be more accurate than those obtained utilizing one-dimensional cost functions and AHP is found to be effective tool to deal with optimal route selection problem.
\end{abstract}

Keywords: optimal path, AHP, MADM, traffic congestion.

\section{Introduction}

Due to rapid urbanization, the tremendous rise in number of vehicles is variably accompanied by ever increasing volume of traffic and intense traffic congestion on roads. Almost every city in India is facing acute traffic problem in regards to delay, congestion, pollution, accidents, parking etc. These problems contribute not only loss of precious manpower but also results in additional fuel consumption, development of mental stress and overall feel bad environment for the driver. Since traffic congestion has been one of the major issues that most of the metropolises are facing, the important task for researcher is to find corrective measure and one of it may be to find shortest path between origin \& destination sources which is not only optimal distant but carry minimum costs for other criterion such as travel time, traffic volume, no. of road junctions, roadside parking, encroachment's along the road etc.

Finding optimal path on congested road in peak hours of urban traffic condition is a complicated and ubiquitous problem. Continuous changes of traffic congestion with respect to the time lead to change the travel times of transportation network. These changes show the importance of time in transportation analyses in addition to the location. So determining the optimal path in

\footnotetext{
${ }^{1}$ Corresponding author: skukadapwar@gpnagpur.ac.in
} 
a time-dependent transportation network is a challenging task. This article proposes the spatial analysis of finding the optimal path between any two specific locations in a road network of the city where traffic condition changes continuously with time.

The route guidance system provides an optimum route to drivers based on a cost function and a route solution. The cost function is related to the distance towards destination, travel time (TT), or the cost of a road segment, etc. The route choice mechanism can provide the optimum route for drivers, based on the cost function. The route selection mechanism is the key technique of vehicle navigation systems providing route-planning strategy for travelers. Defining suitable mathematical models to represent the route selection mechanism in traditional methods uses numerical techniques and methods where perceived traffic attributes are treated as crisp inputs. However, much of human reasoning is based on vague, imprecise, and subjective values. Thus, the traditional methods ignore the presence of vagueness and ambiguity in drivers' perception, making them difficult to be valid mathematical models. Traffic attributes as distance, travel time, traffic volume, encroachment and parking on road, road width and number of intersections were considered for problem of study.

The research paper is organized as follows, Section 2 describes the state of art literature, Section 3 describes the brief overview of MADM method like AHP, Section 4 describes the experimental setup and scenario considered for study, followed by weight estimation AHP and various experimental analysis for different criteria's like shortest path over specified origin \& destination zone using individual attributes and optimal path over specified origin \& destination zone using MADM methods. Last the conclusion and outlook towards future research work is presented in Section 5 .

\section{Literature Review}

Efficient management of traffic network requires, the shortest route from one point (node) to another is known; this is termed as the shortest path. "Optimal" refers to shortest time, shortest distance, or least total cost. Finding the shortest path is an important task in any network and transportation related analyses. This problem arises as a main decision question or as a step in some situation. There are many variations, depending on the type of network and costs involved, and source/ destination pairs of nodes for which we need solution (Rardin, 2003). As per shortest path algorithm by Dijkstra (1959), each node is labeled with its distance from the source node along the best-known path. Initially, no paths are known, so all nodes are labeled with infinity. As the algorithm proceeds and paths are found, the labels may change, reflecting better paths. A label may be tentative or permanent. Initially, all labels are tentative. When it is discovered that a label represents the shortest possible path from the source to node, it is made permanent and never changed thereafter. However, this approach is not feasible for dynamic networks, where the travel cost is time-dependent or randomly varying.

Zhan and Noon (1998) had a comprehensive study of shortest path algorithms on 21 real road networks from 10 different states in the U.S., with networks ranging from $1600 / 500$ to $93000 / 264000$ nodes/arcs. In this study, 
Dijkstra (1959) based algorithms, however differing in data structure; outperform other algorithms in one-to-one or one-toall fastest path problems. Wei et al. (2010) found out shortest path of an OD pair for different departure time by $\mathrm{A}^{*}$ short path algorithm. A case study is carried out by using one day floating data in Wuhan, China. Parbat (2001) generated MPT (minimum path tree) using Moore's algorithm. The travel time study is conducted in Indore city, India using test car technique and MPT from each origin zone to all destination zone were obtained incorporating travel time and distance as factor separately. Ramazani et al. (2010) proposed a method to solve shortest path problems in route choice process when each link travel time is fuzzy no. called as perceived travel time (PTT) which is subjective travel time perceived by a driver. They used FSPA (fuzzy shortest path algorithm) to find shortest path in an urban transportation network.

Realizing the traffic status of real-time road network, the tasks of optimal path selection required to be evaluated by considering different type of criteria i.e. traffic, economic, environmental and social (Nosal and Solecka, 2014). For this purpose during last decades multi-criteria methods came into use and numerous methods have been developed which are classified as multi-criteria analysis methods (e.g. PROMEThEE, ELECTRE, AHP etc.). The multi-criteria analysis method AHP - Analytic Hierarchy Process is widely used as decision making tool in the process of transportation planning (Satty, 1995). AHP has been used for analysing different types of problems in the field of transportation engineering. Nosal and Solecka (2014) presented the idea of travel demand management and basic concepts of urban public transport integration. In order to determine best choice out of different variants the AHP ranking method was used. The practical application of proposed method was demonstrated in the city of Cracow. Pogarcic and Davidovic (2008) analyses possibilities of applying AHP methods in making decision regarding planning and implementation of plans in traffic and ensuring qualitative business logistics. Yedla and Shrestha (2003) in their study tested the group aggregation methods which are commonly employed in AHP against the standard social choice axioms and a comparative analysis has been carried out. Delhi urban transport system was selected as a case in which AHP has been applied to prioritize the selected alternative options for energy efficient and less polluting transport system in Delhi. Dubey et al. (2013) tried the route choice preference using nine route attributes (Distance, Time, Speed, Delay, Pavement condition, Parked vehicle on side of road, Aesthetic, Comfort and familiarity of route). The modelling is done using three techniques, namely multinomial logit model, fuzzy rule based inference system (FIS) and adaptive neurofuzzy inference system (ANFIS). It has been found that all three models have almost $100 \%$ prediction accuracy at aggregate level. $\mathrm{Qu}$ et al. (2008) set up Hybrid Multicriteria Decision Making (MCDM) on the basis of fuzzy analytic hierarchy process (AHP) and artificial neural network (ANN) theory for route selection of multimodal transportation network. Sadeghi-Niaraki et al. (2010) used multidimensional variables combined with multi-dimensional cost model (MCDM) to develop a road network cost function for route finding analysis in Iran. Piantanakulchai and Saengkhao (2003) conducted a study of alternative motorway alignments in Thailand. They 
estimated impacts by the aid of Geographical Information System (GIS) and AHP model developed and the best alignment was proposed by generating a least cost path which is most socially preferable. In India, with our knowledge, application of AHP is found very limited in route selection of multimodal transportation network. This study presented development of AHP model for optimal path finding in an urban transportation network.

\section{Proposed Approach for Optimal Route Selection Using MADM Methods}

Multiple criterion decision making (MCDM) refers to decision making in the presence of multiple, usually conflicting criteria. The MCDM problems can be broadly classified into two categories: multiple attribute decision making (MADM) and multiple objective decision making (MODM), depending on whether the problem is alternative selection problem or a objective problem. The multiple attribute decision making is employed when problem which involves selection from among finite number of alternatives. Alternatives, Attributes, weight or relative importance of each attribute and measure of performance of alternatives with respect to the attributes are the main parts in each decision table of MADM methods (Rao, 2013; Rao, 2007).
MADM methods are generally discrete, with a few numbers of predetermined alternatives. MADM is an approach employed to solve problems involving selection from among a finite number of alternatives. An MADM method specifies how attribute information is to be processed in order to arrive at a choice. Of the many MADM methods reported in the literature (Triantaphyllou, 2000; Hwang and Yoon, 1981), we have applied AHP method to solve optimal route selection problem.

\subsection{AHP (Analytical Hierarchy Processing)}

Analytic hierarchy process (AHP) is one of the most popular analytical techniques for solving complex decision making problems (Satty, 1995; Saaty, 1980). A number of functional characteristics make AHP a useful methodology.

These include the ability to handle decision situations involving subjective judgments, multiple decision makers, and the ability to provide measures of consistency of preferences (Triantaphyllou, 2000). Designed to reflect the way people actually think, AHP continues to be the most highly regarded and widely used decision making method. AHP can efficiently deal with objective as well as subjective attributes.

Step 1: Compute the decision matrix

Table 1

Decision Matrix Table in MADM Methods

\begin{tabular}{l|llllll}
\hline \multirow{2}{*}{ Alternatives } & \multicolumn{5}{l}{ Attributes (weights) } \\
\cline { 2 - 7 } $\mathrm{A}_{1}$ & $\mathrm{~B}_{1}\left(\mathrm{w}_{1}\right)$ & $\mathrm{B}_{2}\left(\mathrm{w}_{2}\right)$ & $\mathrm{B}_{3}\left(\mathrm{w}_{3}\right)$ & - & - & $\mathrm{B}_{\mathrm{m}}\left(\mathrm{w}_{\mathrm{m}}\right)$ \\
$\mathrm{A}_{2}$ & $\mathrm{C}_{11}$ & $\mathrm{C}_{12}$ & $\mathrm{C}_{13}$ & - & - & $\mathrm{C}_{14}$ \\
$\mathrm{~A}_{3}$ & $\mathrm{C}_{21}$ & $\mathrm{C}_{22}$ & $\mathrm{C}_{23}$ & - & - & $\mathrm{C}_{24}$ \\
- & $\mathrm{C}_{31}$ & $\mathrm{C}_{32}$ & $\mathrm{C}_{33}$ & - & - & $\mathrm{C}_{34}$ \\
- & - & - & - & - & - & - \\
$\mathrm{A}_{\mathrm{n}}$ & - & - & - & - & - & - \\
\hline
\end{tabular}


The decision table, given in Table 1 , shows alternatives, $A_{i}$ (for $\left.i=1,2, \ldots, n\right)$, attributes, $B_{j}($ for $j=1,2, \ldots, m)$, weights of attributes, $w_{j}($ for $j=1,2, \ldots, m)$ and the measures of performance of alternatives, $C_{i j}$ (for $i=1,2$, $\ldots, n ; j=1,2, \ldots, m)$. Given multi attribute decision making method and the decision table information, the task of the decision maker is to find the best alternative and/ or to rank the entire set of alternatives. To consider all possible attributes in decision problem, the elements in the decision table must be normalized to the same units.

Step 2: Compute the normalized decision matrix: The attributes can be considered as beneficial or non-beneficial. Normalized values are calculated by $\left(C_{i j}\right)_{K} /\left(C_{i j}\right)_{L}$, where $\left(C_{i j}\right)_{K}$ is the measure of the attribute for the $K^{\text {th }}$ alternative, and $\left(C_{i j}\right)_{L}$ is the measure of the attribute for the $L^{\text {th }}$ alternative that has the highest measure of the attribute out of all alternatives considered. This ratio is valid for beneficial attributes only. A beneficial attribute (e.g., efficiency) means its higher measures are more desirable for the given decision-making problem. By contrast, non-beneficial attribute (e.g., cost) is that for which the lower measures are desirable, and the normalized values are calculated by $\left(C_{i j}\right)_{L} /\left(C_{i j}\right)_{K}$.

Step 3: Assuming $M$ attributes, the pair-wise comparison of attribute $i$ with attribute $j$ yields a square matrix $B_{M * M}$ where $b_{i j}$ denotes the comparative importance of attribute $i$ with respect to attribute $j$. In the matrix, $b_{i j}=$ 1 when $i=j$ and $b_{j i}=1 / b_{i j}$. The judgments are entered using the fundamental scale of the analytic hierarchy process (Triantaphyllou, 2000; Saaty, 1980).

\section{Table 2}

Saaty's 1-9 Scale of Pair Wise Comparison

\begin{tabular}{ll}
\hline Intensity of importance & Definition \\
\hline 1 & Equal importance \\
3 & Moderate importance \\
5 & Strong importance \\
7 & Very strong importance \\
9 & Extreme importance \\
2468 & Intermediate values \\
\hline
\end{tabular}

$B_{M * M}=\left[\begin{array}{ccccc}1 & b 12 & b 13 & - & b 1 M \\ b 21 & 1 & b 23 & - & b 2 M \\ b M 1 & b M 2 & b M 3 & - & 1\end{array}\right] \quad w_{j}=\frac{G M_{j}}{\sum_{j}^{M} G M_{j}}$

Find the relative normalized weight $\left(w_{j}\right)$ of each attribute by (a) calculating the geometric mean of the $i$-th row, and (b) normalizing the geometric means of rows in the comparison matrix. This can be represented as:

$G M_{j}=\left[\prod_{j=1}^{M} b_{i j}\right]^{1 / M}$
The geometric mean method of AHP is commonly used to determine the relative normalized weights of the attributes, because of its simplicity, easy determination of the maximum Eigen value, and reduction in inconsistency of judgments.

a. Calculate matrices $\mathrm{A} 3$ and $\mathrm{A} 4$ such that $\mathrm{A} 3=\mathrm{A} 1{ }^{*} \mathrm{~A} 2$ and $\mathrm{A} 4=\mathrm{A} 3 / \mathrm{A} 2$, where 


\author{
$\mathrm{A} 2=\left[w_{1}, w_{2}, \ldots . ., w_{j}\right] T$. where $\mathrm{A} 1$ is \\ relative importance matrix.
}

b. Determine the maximum Eigen value $\lambda \max$ that is the average of matrix $\mathrm{A} 4$.

c. Calculate the consistency index $\mathrm{CI}=$ $(\lambda \max -M) /(M-1)$. The smaller the value of $\mathrm{CI}$, the smaller is the deviation from the consistency.

d. Obtain the random index (RI) for the number of attributes used in decision making. Refer to Table 3 for details.

Table 3

Random Index (RI) Values

\begin{tabular}{|l|l|l|l|l|l|l|l|l|}
\hline Attributes & 3 & 4 & 5 & 6 & 7 & 8 & 9 & 10 \\
\hline RI & 0.52 & 0.89 & 1.11 & 1.25 & 1.35 & 1.4 & 1.45 & 1.49 \\
\hline
\end{tabular}

a. Calculate the consistency ratio $\mathrm{CR}=$ CI/RI. Usually, a CR of 0.1 or less is considered as acceptable, and it reflects an informed judgment attributable to the knowledge of the analyst regarding the problem under study.

Step 4: The next step is to obtain the overall or composite performance scores for the alternatives by multiplying the relative normalized weight $\left(w_{j}\right)$ of each attribute (obtained in step 3) with its corresponding normalized weight value for each alternative (obtained in step 2), and summing over the attributes for each alternative is computed as Eq. (3):

$P_{i}=\sum_{j=1}^{m} w_{j}\left(C_{i j}\right)_{\text {normal }}$

Where $\left(C_{i j}\right)$ normal represents the normalized value of $C_{i j}$, and $P_{i}$ is the overall or composite score of the alternative $A_{i}$. The alternative with the highest value of $P_{i}$ is considered as the best alternative.

\section{Selection of Study Area}

The study area selected for performing the present research comprises Nagpur city, the second capital of Maharashtra state and major administrative, commercial, medical and educational center of central India. The city is experiencing the common traffic problems as most of cities of developing countries are facing i.e. rapid increase in number of vehicles, increased traffic volume compared to capacity of the road, increase in number of trips, heterogeneous traffic, roadway parking, mixed land-use along the road, encroachments and pedestrian movement on road etc.

The heavy migration of people from all the parts of country is aggregating the traffic problem. It is estimated that population of city will be about 40 lacks by 2021 .

The study area selected is $217.56 \mathrm{sq} . \mathrm{km}$ located within municipal corporation boundary. The road network of Nagpur city is divided in 10 zones comprises of 72 wards designated as number 1 to 72 consist of nodes and links.

Total intersection nodes selected for study are 166 and numbered from 73 to 238 . There are 215 actual links joining intersection nodes. Coded road network is shown in Fig. 1. 


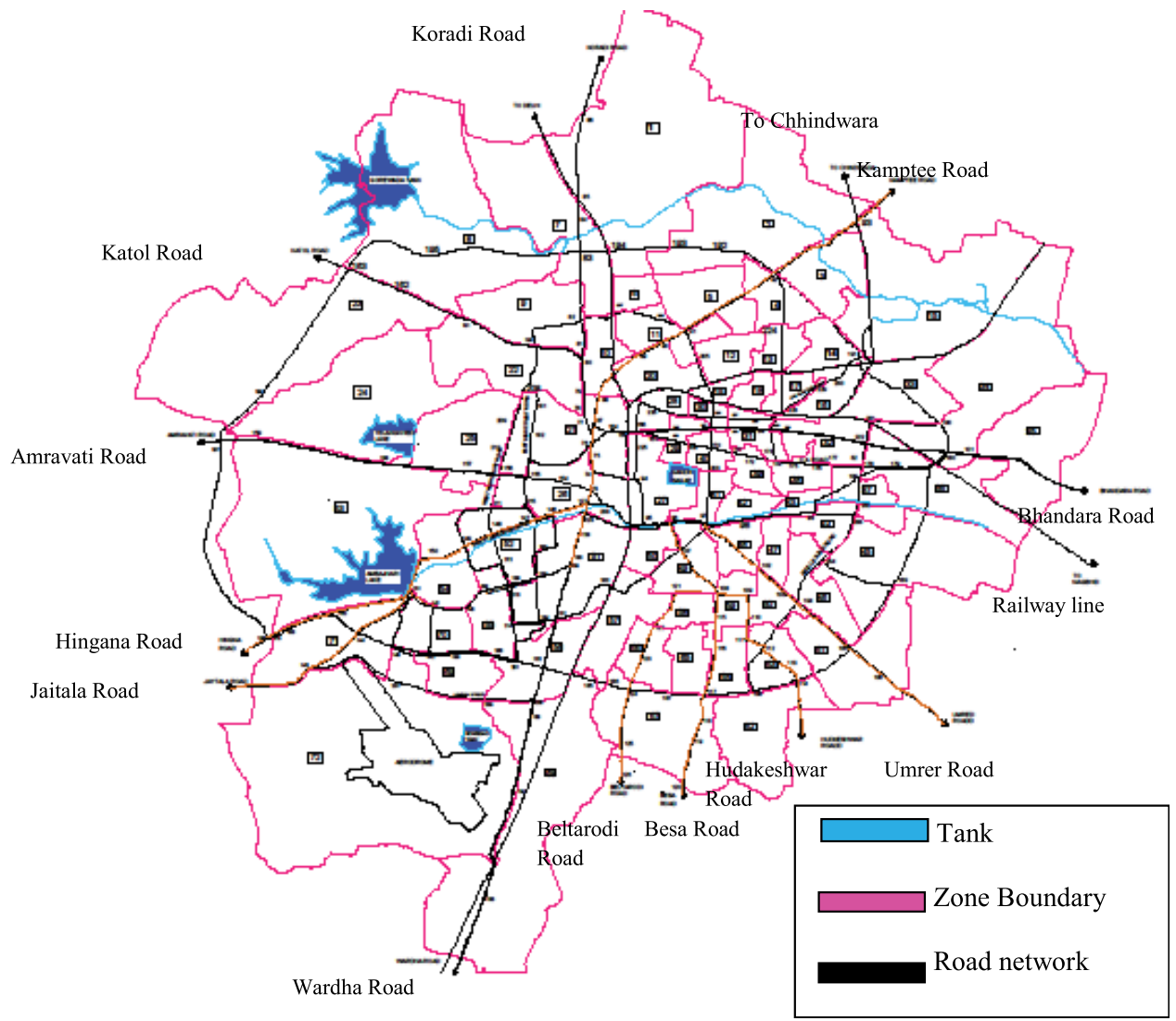

Fig. 1.

Road Network of Nagpur City

\subsection{Traffic Field Study and Surveys}

The field work is carried out invariably under perfect weather condition on normal working day in the month of January-February 2014. Field work is carried out in two parts: (A) Travel time study and (B) Traffic volume count.

For conducting travel time study, the test car technique is adopted. Test car was run on major traffic links in peak hours and non- peak hours. The average speed, travel time and distance between intersections was observed and tabulated. The timing of field work was from $9.30 \mathrm{am}$ to $11.30 \mathrm{am}$ during morning peak hour and $5.30 \mathrm{pm}$ to $7.30 \mathrm{pm}$ during evening peak hours. Test car was made to run on road network between 7 am to 9 am and 12 noon to 5 pm to record travel time in non-peak hours. During the study, the test car was run at speed which in the opinion of driver is the representative of average speed of all the vehicles in stream of flow at the time of run.

The traffic volume data was collected manually by team of experts during the peak 
hours of the day. Number of vehicles passing over the total 215 no. of links was noted in to and fro direction separately in designed tally sheet.

In addition to above, detailed surveys were conducted for collecting primary information regarding road width, number of intersections, parking and encroachment along roadway etc.

\subsection{Weight Estimation Using AHP}

In this section weight estimation using AHP is provided, where first dependency matrix is created based on Saaty's scale. Referring to matrix A1, every attribute is compared with others, ex. DT (Distance) first compared with itself so value is 1 , then $\mathrm{DT}$ is compared with TT (Travel Time) as in this case TT is moderately important than DT is value will be $1 / 3$, when DT is compared with PCU (Traffic Volume), in this case DT is moderately important than PCU thus its value will be 3 . Accordingly weight estimation is carried out by knowing the importance of individual attribute in real transportation network.

$$
\left.\mathrm{A} 1=\begin{array}{c|ccccccc}
\mathrm{DT} & 1 & 1 / 3 & 3 & 4 & 4 & 4 & 3 \\
\mathrm{TT} & 3 & 1 & 3 & 5 & 5 & 5 & 2 \\
\mathrm{PCU} & 1 / 3 & 1 / 3 & 1 & 2 & 2 & 3 & 1 / 3 \\
\mathrm{RW} & 1 / 4 & 1 / 5 & 1 / 2 & 1 & 2 & 3 & 1 / 3 \\
\mathrm{NI} & 1 / 4 & 1 / 5 & 1 / 2 & 1 / 2 & 1 & 1 / 3 & 1 / 4 \\
\mathrm{PR} & 1 / 4 & 1 / 5 & 1 / 3 & 1 / 3 & 3 & 1 & 1 / 5 \\
\mathrm{ENR} & 1 / 3 & 1 / 2 & 3 & 3 & 4 & 5 & 1
\end{array}\right]
$$

Where DT is Travel Distance, TT is Travel Time, PCU is Traffic Volume in Passenger Car Unit, RW is Road Width, NI is Number of Intersections, PR is Parking on Road and ENR is Encroachment along road.



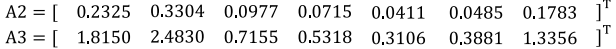
$\mathrm{A} 4=\left[\begin{array}{lllllll}7.8076 & 7.5142 & 7.3261 & 7.4400 & 7.5570 & 7.9957 & 7.4905\end{array}\right]$ $\lambda \max =7.5902$

$\mathrm{CI}=0.0984$

$\mathrm{CR}=0.0729$

\subsection{Shortest Path between Specified Zones}

Dijkstra's algorithm solves the single-source shortest-path problem when all edges have non-negative weights. Let $G=\{V, E\}$ be a directed weighted graph with $V$ having the set of vertices. The special vertex $s$ in $V$, where $s$ is the source and let for any edge $e$ in $E$, Edge Cost(e) be the length of edge $e$. For the weighted directed graph its adjacency matrix $A=\left(a_{i j}\right)_{n \times n}$ is defined as Eq. (4):

$a_{i j}=\left\{\begin{array}{c}w_{i j},\left(V_{i}, V_{j}\right) \in E \\ \infty,\left(V_{i}, V_{j}\right) \notin E\end{array} \mathrm{i}, j=1,2, \ldots n\right.$

where $W_{i j}$ denotes the weight of $\operatorname{arc}\left\langle V_{i}, V_{j}>\right.$, $\infty$ denotes that there is no edge between $V_{i}$ and $V_{j}$ (Dijkstra, 1959).

The main steps of the Dijkstra's algorithm are as follows (Dijkstra, 1959),

1. Use adjacency matrix $C$ to store network information. $C_{i j}$ denotes the weight of $\operatorname{arc}\left\langle V_{i}, V_{j}\right\rangle$. If there is no arc between $V_{i}$ and $V_{j}$, then $C_{i j}$ is set to $\infty . d_{i}$ is defined as the weight from the source points to node $V_{i}$. Initialize starting point as $d_{s}$ $=0$ and $D_{i}=s i$.

2. Select $V_{p}$, then we have,

$d_{p}=\min \left\{d_{p} \mid v_{p} \in V-S\right\}$

$V_{p}$ is the end point in the shortest path starting from the source point $V_{s}$. Then,

$S=S \cup\left\{v_{i}\right\}$ 
Set the end point to $V_{t}$. If $V_{p}$ is equal to $V_{t}$, $d_{p}=\min \left[d_{i}, d_{k}+l_{k p}\right], v_{p} \in V-S, V_{k} \in S$ which means $d_{p}$ is the shortest path from starting point $V_{s}$ to end point $V_{t}$, then algorithm stops. Otherwise, turn to step 3.

where $l_{k p}$ is the direct distance from point $k$ to point $j$.

3. Modify the length of the shortest path from $V_{s}$ to any point $V_{t}$ in set of $(V-S)$, and sadisfy $d_{p}$ as follow:

4. Repeat step 2 and step 3, until the shortest path is found from starting point $V_{s}$ to end point $V_{t}$.

Table 4

Shortest Path over Specified Zones

\begin{tabular}{ll}
\hline Criteria for shortest path & Path (Intersection Node No.) \\
\hline $\begin{array}{l}\text { Source Zone: } 4 \\
\text { Destination Zone: } 69\end{array}$ & \\
\hline Distance & 42242232282272041702039710720213113220120069 \\
Travel Time & 49291908988877675747312712812913013613113220120069 \\
PCU & 4921921931948319516319619719815315214415114114269 \\
Road width & 49219119018018910511418811718712418618513220120069 \\
Parking on road & 4921921931948319516319619719815315214414314269 \\
Encroachment & 4921921931948319516319619719815315215114114269 \\
\hline
\end{tabular}

Discussion: Table 4 describes the shortest path obtained for individual traffic attribute criteria's like Travel Distance, Travel Time, Traffic Volume in PCU, Road Width, Parking on Road, Encroachment along link for source zone no. 4 to destination zone no. 69. The six individual paths are obtained w.r.t. respective attribute as shown in Fig. 2 using Dijkstra's algorithm. AHP is modeled to get best path out of six identified paths.

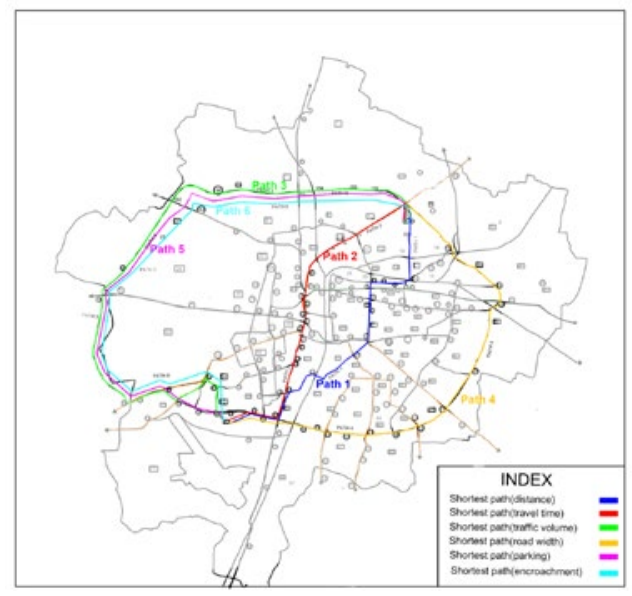

Fig. 2.

Shortest Path for Individual Traffic Attribute 


\subsection{Optimal Path Using AHP}

After obtaining shortest path between two specified zones (Zone No. 4-69) w.r.t. individual seven attribute using
Dijkstra's algorithm, the question of complex decision making comes into action thus Analytic Hierarchy Process (AHP) is modeled for choosing the best suited path.

\section{Table 5}

Optimal Path over Specified Intersections Using AHP

\begin{tabular}{|c|c|c|c|c|c|c|c|c|c|}
\hline \multicolumn{10}{|c|}{ Source Zone: 4 Destination Zone: 69} \\
\hline \multirow[b]{2}{*}{ Alternatives } & \multicolumn{7}{|c|}{ Attributes } & \multicolumn{2}{|l|}{ AHP } \\
\hline & DT & TT & PCU & RW & NI & PR & ENR & Score & Rank \\
\hline Path1 & 11.6 & 2289 & 24156.15 & 1.938024 & 14 & 44 & 40 & 0.8179 & 1 \\
\hline Path2 & 12.24 & 1540 & 48624.5 & 1.557578 & 19 & 62 & 59 & 0.7939 & 2 \\
\hline Path3 & 23.8 & 3023 & 20966.27 & 1.44524 & 16 & 30 & 32 & 0.6827 & 5 \\
\hline Path4 & 18.3 & 2437 & 34443.9 & 1.000002 & 16 & 46 & 43 & 0.6435 & 6 \\
\hline Path5 & 22.4 & 2879 & 21380.6 & 1.273018 & 15 & 29 & 31 & 0.6993 & 3 \\
\hline Path6 & 23.4 & 2969 & 22520.47 & 1.378573 & 15 & 29 & 30 & 0.6936 & 4 \\
\hline
\end{tabular}

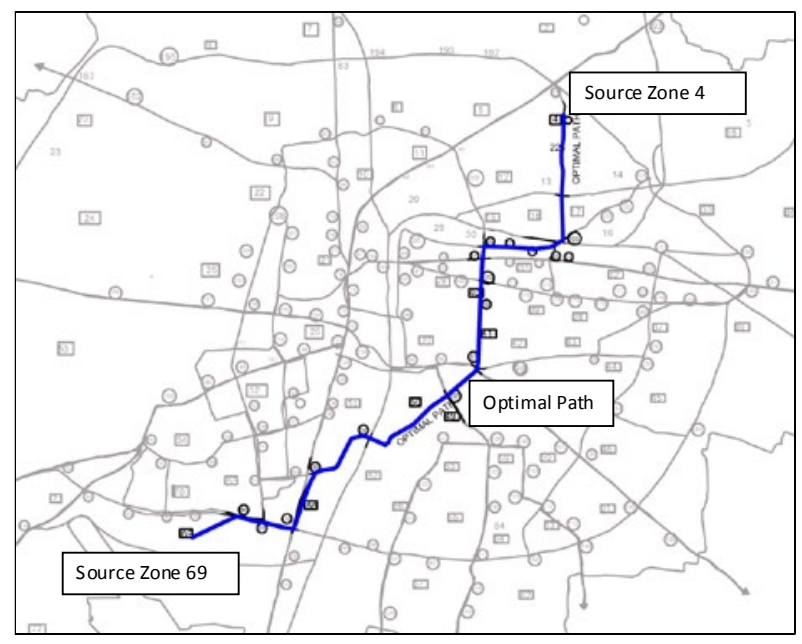

Fig. 3.

Diagram of Optimal Path Obtained Using AHP

Discussion: Table 5 describes the optimal path obtained by considering all seven traffic attributes. AHP is modeled to obtain the optimal path. Paths which are obtained in
Table 4 for specific source and destination are provided as alternatives to AHP and seven traffic attributes namely distance, travel time, traffic volume in PCU, road 
width, no. of intersection, parking along road and encroachment on road are considered. Table 5 provides the corresponding score for individual alternative (path) and ranking, the path which is having highest score is considered as optimal path. Fig. 3 shows the optimal path obtained between zone no. 4 to zone no. 69. In this case Path 1 is ranked as optimal path as the score of this path using AHP is found highest thus the said path may be selected as most suitable path considering the traffic condition in real traffic network between specified zones.

\section{Conclusion}

A useful routing system should have the capability to support the driver effectively in deciding on an optimum route to his/her preference. In this research paper, shortest path over specified zones using individual traffic attribute criteria's like travel distance, travel time, traffic volume (PCU), road width, number of intersections, parking on road, encroachment are considered and also AHP is modeled to obtain the optimal path by considering above mentioned traffic attributes over specified zones. Using this model, optimal path between various zones of Nagpur city is found out. An example for source zone no. 4 to destination zone no. 69 is illustrated in the paper.

For the present case study, the Consistency Ratio obtained for AHP is 0.0729 which is much less than 0.1, thus optimal paths obtained using AHP for specified origin $\&$ destination zones are valid. From the experimental results for optimal path over specified zones, it is observed that in most cases the optimal path is prominently obtained for travel distance or travel time as evaluation criteria. This methodology paves the way for more intelligent traffic system.

\subsection{Concluding Remarks}

1. AHP is applied to route decision making process. Relative importance of each attribute in AHP is modeled. A case study of real time traffic network in Nagpur, India was conducted during peak hour period. Impacts were estimated by the aid of detailed data collected during real time traffic condition by expert team and AHP model developed.

Composite weighted AHP scored were used to generate AHP decision surface. Finally, the best decision was proposed by generating a least cost path which is most socially preferable.

2. Each objective could be represented by attributes which are either quantifiable or unquantifiable. In this study, the relative importance of quantifiable attributes such as the travel distance, travel time, traffic volume, road width, no. of intersections etc., were modeled using their relative level to standards.

For the relative importance of unquantifiable attributes such as parking condition and encroachments on road AHP was applied.

Recommendations for further studies are:

1. This study applied maximum total social benefits as a decision rule. However, applying different decision criteria may result in different solution.

2. The characteristic of response function of various impacts should be further studied.

3. Number of roads in traffic network is required to be increased to get more realistic results. 


\section{References}

Dijkstra, E.W. 1959. A note on two problems in connexion with graphs, Numerische Mathematik, Springer, 1(1): 269-271.

Dubey, S.K.; Mishra, D.; Arkatkar, S.S.; Singh, A.P.; Sarkar, A.K. 2013. Route Choice Modelling Using Fuzzy logic and Adaptive Neuro-fuzzy, Modern Traffic and Transportation Engineering Research, 2(4): 11-19.

Hwang, C.L.; Yoon, K.P. 1981. Multiple attribute decision making: methods and applications, Springer. 225p.

Nosal, K.; Solecka, K. 2014. Application of AHP Method for Multi-criteria Evaluation of Variants of the Integration of Urban Public Transport, Transportation Research Procedia, 3: 269-278.

Parbat, D.K. 2001. Development of O-D Time-Distant Plot and Isochron Map for Indore city, Indian Highways, 29(9): 17-27.

Piantanakulchai, M.; Saengkhao, N. 2003. Evaluation of alternatives in transportation planning using multistakeholders multi-objectives AHP modeling. In Proceedings of the Eastern Asia Society for transportation studies, 1613-1628.

Pogarčić, I.; Davidović, V. 2008. Application of AHP method in traffic planning. In Proceedings of the 16th International Symposium on Electronics in Traffic.

Qu, L.; Chen, Y.; Mu, X. 2008. A Transport Mode Selection Method for Multimodal Transportation Based on an Adaptive ANN System. In Proceedings of the Natural Computation, ICNC '08. Fourth International Conference, vol. 3: 436-440.

Ramazani, H.; Shafahi, Y.; Seyedabrishami, S.E. 2010. A Shortest Path Problem in an Urban Transportation Network Based on Driver Perceived Travel Time, Transaction A: Civil Engineering, 17(4): 285-296.
Rao, R. 2007. Decision Making in the Manufacturing Environment Using Graph Theory and Fuzzy Multiple Attribute Decision Making, Springer Series in Advanced Manufacturing. $371 \mathrm{p}$.

Rao, R. 2013. Decision Making in Manufacturing Environment Using Graph Theory and Fuzzy Multiple Attribute Decision Making Methods, Springer Series in Advanced Manufacturing. $291 \mathrm{p}$.

Rardin, R.L. 2003. Optimization in Operations Research, Pearson Education, Delhi, India.

Saaty, T.L. 1980. The analytic hierarchy process, planning, priority setting, resource allocation, New York: Mc Graw Hill.

Sadeghi-Niaraki, A.; Kim, K.; Varshosaz, M. 2010. Multi-Criteria Decision-based Model for Road Network Process, International Journal of Environmental Research, 4(4): 573-582.

Satty, T.L. 1995. Transport Planning with Multiple Criteria: The Analytic Hierarchy Process Applications and Progress Review, Journal of Advanced Transportation, 29(1): 81-126.

Triantaphyllou, E. 2000. Multi-criteria decision making methods: a comparative study, Springer. $265 \mathrm{p}$.

Wei, T.; Zhixiang, F.; Qingquan, L. 2010. Exploring time varying shortest path of urban OD Pairs based on floating car data. In Proceedings of the 18th International Conference on Geoinformatics, 1-6.

Yedla, S.; Shrestha, R.M. 2003. Multi-criteria approach for the selection of alternative options for environmentally sustainable transport system in Delhi, Transportation Research Part A: Policy and Practice, 37(8): 717-729.

Zhan, F.B.; Noon, C.E. 1998. Shortest Path Algorithms: An Evaluation Using Real Road Networks, Transportation Science, 32(1): 65-73.

\section{jitte 24}

